\title{
Two-dimensional crossover and strong coupling of plasmon excitations in arrays of one-dimensional atomic wires
}

\author{
T. Lichtenstein, ${ }^{1}$ J. Aulbach, ${ }^{2}$ J. Schäfer, ${ }^{2}$ R. Claessen, ${ }^{2}$ C. Tegenkamp, ${ }^{1,3}$ and H. Pfnür ${ }^{1,3, *}$ \\ ${ }^{1}$ Institut für Festkörperphysik, Leibniz. Universität Hannover, Appelstraße 2, 30167 Hannover, Germany \\ ${ }^{2}$ Physikalisches Institut and RCCM, Universität Würzburg, Am Hubland, Würzburg, Germany \\ ${ }^{3}$ Laboratorium für Nano- und Quantenengineering (LNQE), Leibniz Universität Hannover, \\ Schneiderberg 39, 30167 Hannover, Germany \\ (Received 6 November 2015; revised manuscript received 30 March 2016; published 18 April 2016)
}

\begin{abstract}
Dimensional crossover is of high relevance to understanding real-world quasi-one-dimensional (1D) systems. Here we study the collective excitations, measured as plasmon dispersions in an electron energy loss experiment, in a tunable family of model systems, namely, Au chains on stepped $\mathrm{Si}(h h k)$ substrates, that allow variations of chain widths and interchain spacings. We indeed observe 1D-like dispersions, but with a significant influence of higher dimensions. Surprisingly, we find that it is not the interchain coupling but the width of the conduction channel, as confirmed by tunneling spectroscopy, that dominates the excitations.
\end{abstract}

DOI: 10.1103/PhysRevB.93.161408

One-dimensional (1D) electronic systems have highly attractive properties such as quantization of conductance, extremes of electronic correlation manifested by spin-charge separation, charge and spin density waves [1,2], triplet superconductivity, and Luttinger liquid behavior [3-5]. Due to their inherent instability, however, structural embedding and understanding of the coupling to other dimensions is of supreme importance. Indeed, many 1D properties can still be observed in these quasi-1D systems [6-10]. In addition, these systems host a variety of instabilities with a wealth of associated phase transitions [11]. On the other hand, the excitation spectra of these systems and their dynamics are still largely unexplored, which is particularly true for collectively excited plasmonic states.

Apart from these fundamental aspects, plasmons play an important role, e.g., in sensor technology [12], improvement of quantum efficiency in photovoltaic devices [13], and even in cancer research [14]. A new field of plasmon research has been opened recently by collective excitations of low-dimensional electron gases, called sheet plasmons $[15,16]$, which have wavelengths that are typically three orders of magnitude shorter compared to photons of the same frequency. Thus $\mathrm{THz}$ plasmonics on the scale of a few nanometers becomes feasible.

One-dimensional (1D) metallic wires and their plasmonic excitations would be ideal for directed energy transport on the nanoscale, since quasilinear dispersion is predicted, at least in the long wavelength limit [17], for these 1D plasmons. Such dispersions have indeed been found for regular arrays of atomic wires on insulating substrates [18-20]. Moreover, confinement effects in these metallic subunits on the surface lead to the formation of intersubband excitations [19-21].

Before realizing such visions, several fundamental properties of these quasi-1D plasmons need to be clarified, comprising, in particular, the question of dimensional crossover, but also the correct treatment of many-body effects, electronic correlations, and Coulomb screening [22-24]. These open topics led to a partly unsatisfactory description of experimental results in the past $[18,19,25]$.

\footnotetext{
*pfnuer@fkp.uni-hannover.de
}

To address such fundamental aspects for 1D and 2D systems, the growth of various metals in the submonolayer regime on semiconducting surfaces provides a superb approach. The adsorbate induced band structure is generally electronically decoupled from the bulk bands of the host material. In particular, Au chains on regularly stepped Si(111) surfaces at various tilt angles towards the [ $\overline{1} \overline{1} 2]$ direction offer a unique opportunity to modify physical properties in a controlled fashion, not provided by other atomic wire systems [26,27]. Depending on coverage and vicinality, the widths of the $\mathrm{Au}$ chains and their interwire spacing can be tuned, while their electronic band structures are still very similar. So, 0.2 monolayers (ML) of $\mathrm{Au}$ on $\mathrm{Si}(557)$, e.g., result in the growth of single atom $\mathrm{Au}$ chains and a row of $\mathrm{Si}$ adatoms on each miniterrace with an interwire spacing of $19.2 \AA[27,28]$. In contrast, $\mathrm{Si}(553)$ and $\mathrm{Si}(775)-\mathrm{Au}$ host double $\mathrm{Au}$ chains in the center of the terrace [26,29]. The interwire spacing is $14.8 \AA$ for $\operatorname{Si}(553)$ and $21.3 \AA$ for $\operatorname{Si}(775)$ [8,27,30]. For double Au chains, nominal coverages of $0.48 \mathrm{ML}$ on $\mathrm{Si}(553)$ and of $0.32 \mathrm{ML}$ on $\mathrm{Si}(775)$ result. Common to all these structures is a graphitic Si honeycomb chain located at the step edges [8,27,30], which-under certain circumstances-can host ordered chains of local magnetic moments $[8,9,26]$.

Each of these systems is characterized by metallic bands that are well known from angle-resolved photoemission spectroscopy (ARPES) measurements [27]. They only disperse along the chain direction $k_{\|}$, and have their minima at the zone boundary. Thus, also the (equilibrium) electron density available for plasmonic excitations is well known.

In fact, these systems locally form the narrowest possible $1 \mathrm{D}$ objects that can be realized, namely, chains that are one or two atoms wide. Therefore, we address here the question of local confinement and dimensionality both for the ground state close to the Fermi level and for the collective excited plasmon state with emphasis on the plasmons. We compare the collective excitations in $\mathrm{Si}(553)-\mathrm{Au}$ and $\mathrm{Si}(775)-\mathrm{Au}$, which have the same structural motif of the double gold chain, with the $\mathrm{Si}(557)-\mathrm{Au}$ system with only a single gold chain. Since the same substrate $(\mathrm{Si})$ is used, we are thus able to concentrate on the influence of structural elements on dispersion and to compare pairs of either identical structural motifs (double gold 
chains) on terraces of a different lateral width, or pairs with a different structural motif (single versus douÂ-ble chains) on terraces with almost identical width.

Although purely $1 \mathrm{D}$ dispersion along the chain direction is found, the lateral extension of the charge distribution turns out to explicitly influence the slope of the measured plasmon dispersion curves. In other words, this crossover into the second (or third) dimension is crucial for this quasi-1D phenomenon, whereas the plasmonic coupling between the wires in the ordered arrays, which is another aspect of dimensional crossover, can in fact be treated as a correction [31,32].

All experiments were performed in two different ultrahigh vacuum chambers operating at a base pressure of $5 \times 10^{-11}$ mbar. One system hosts a high resolution spot profile analysis low-energy electron diffractometer (SPALEED) to investigate and control the sample quality, and a combination of an electron energy loss spectrometer with a LEED diffractometer providing high resolution both in energy and momentum [33] in order to determine plasmon dispersion relations. The overall sample quality was checked by a SPALEED. The vicinal Si substrates ( $\rho \approx 0.01 \Omega \mathrm{cm}, n$ type) were annealed at $1250{ }^{\circ} \mathrm{C}$ for a few seconds, followed by a rapid cooldown. The appropriate coverages of $0.48 \mathrm{ML}$ for $\mathrm{Si}$ (553)$\mathrm{Au}$ and 0.32 ML for Si(775)-Au were evaporated from a gold pearl on a tungsten filament by direct current heating, or from a crucible at a substrate temperature of $630^{\circ} \mathrm{C}$. The coverage has been controlled and calibrated by quartz microbalances placed at the position of the samples [34]. After Au deposition and cooling to room temperature, the samples were quickly annealed to $930^{\circ} \mathrm{C}$ for $<1 \mathrm{~s}$, followed by instantaneous cooling to room temperature. The loss measurements were carried out directly after this postannealing step in order to avoid any influence of residual gas on the surface and electronic structure. The scanning tunneling microscopy (STM) measurements were performed at $77 \mathrm{~K}$ in the second chamber using a low-temperature STM manufactured by Omicron. The overall sample quality here was checked with an optical LEED.

Here, we demonstrate the close structural similarities between the $\mathrm{Si}(553)-\mathrm{Au}$ and $\mathrm{Si}(775)-\mathrm{Au}$ systems which complement and fit to the very similar electronic structures mentioned above. The LEED patterns right after preparation are shown in Fig. 1(a). These patterns are characteristic for regularly stepped (553) and (775) surfaces. They consist of (111)-oriented terraces and steps of double atomic height $(d=$ $3.14 \AA$ ). From the spot splitting of $22 \%$ surface Brillouin zone (SBZ) for $\mathrm{Si}(553)-\mathrm{Au}$ and $15.6 \% \mathrm{SBZ}$ for $\mathrm{Si}(775)$ we derive average terrace widths of $14.8 \AA$ for the $\operatorname{Si}(553)$ and of $21.3 \AA$ for the $\mathrm{Si}(775)$ surface. Thus, the adsorption of $0.48 \mathrm{ML}$ of $\mathrm{Au}$ on $\mathrm{Si}(553)-0.32 \mathrm{ML}$ on $\mathrm{Si}(775)$ - leaves the periodic array of double steps with sharp spots and $\times 2$ streaks unchanged, indicating high quality 1D order. This is in agreement with STM, exemplarily shown for the (775) system in Fig. 1(c). A narrow distribution of terrace widths can be concluded from $\left(k_{\|}, k_{\perp}\right)$ plots in LEED (not shown) and from STM.

High resolution STM, shown in Fig. 1(b) for the $\mathrm{Si}(775)-\mathrm{Au}$ system, reveals details of the atomic arrangement [for an extended set of STM data on Si(553)-Au, see Ref. [9]]. Each terrace hosts three structural motifs. Their origin could be disentangled by a detailed analysis via STM and density functional theory, which is published elsewhere [26]. However, (a)
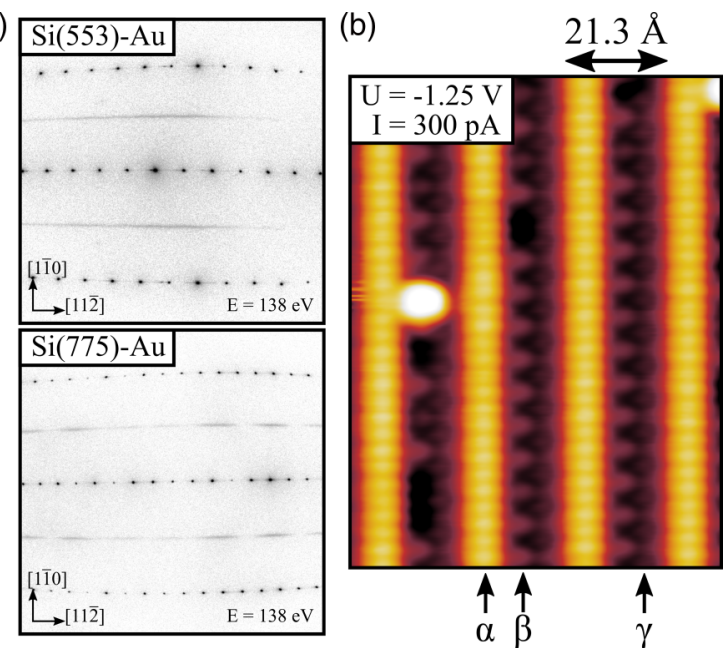

FIG. 1. (a) LEED patterns of the chain structures of $\mathrm{Si}(553)-\mathrm{Au}$ and $\mathrm{Si}(775)-\mathrm{Au}$. (b) STM image of the $\mathrm{Si}(775)-\mathrm{Au}$ chain structure (tunneling conditions $U=-1.25 \mathrm{~V}, I=300 \mathrm{pA}$ ): $\alpha, \beta, \gamma$ mark the $\mathrm{Si}$ terrace edge, a row of $\mathrm{Si}$ adatoms accompanied by $\mathrm{Si}$ restatoms, and a Au double chain with $\times 2$ period doubling, respectively [26]. STM experiments were performed at $77 \mathrm{~K}$, and LEED at $300 \mathrm{~K}$. Bright protrusion could be identified as an adatom defect [26].

we will list our results here: The motif denoted by $\alpha$ could be ascribed to the Si honeycomb chain at the step edge. Chain $\beta$ is formed by a $\mathrm{Si}$ adatom row accompanied by $\mathrm{Si}$ restatoms (i.e., unpassivated Si atoms). Motif $\gamma$, most important for this work, could be identified as a double Au strand [26], similar to what was found in $\mathrm{Si}(553)-\mathrm{Au}[8,9,29]$.

Most importantly, the chain structures show a $2 a=7.7 \AA$ periodicity along the wire direction. Correspondingly, LEED reveals modulated $\times 2$ diffraction streaks. The streaks along the [11 12$]$ direction are indicative for only short-range correlations between the double periodicity along the chains on the different terraces. For $\mathrm{Si}(553)-\mathrm{Au}$, only the $\mathrm{Au}$ chains show the $\times 2$ periodicity. Therefore, the modulation and intensity of the $\times 2$ diffractions streaks there are weaker.

On these well ordered arrays of 1D atomic chains, angleresolved electron energy loss spectroscopy measurements were performed. Figure 2(a) shows sequences of loss spectra on a semilog scale as a function of increasing $k_{\|}$for $\mathrm{Si}(553)$ Au. A similar plot for the orthogonal direction is shown in Fig. 2(b). Corresponding spectra for Si(775)-Au are shown in the Supplemental Material [35]. Close to $k=0$ the spectra are structureless, apart from a small nondispersing feature that dies out quickly with increasing $k_{\perp}$ [dashed line in Fig. 2(b)]. The exponentially decaying loss intensity as a function of loss energy elastic peak, known as the Drude tail, is the typical signature of the continuum of low-energy excitations in metallic systems [36], i.e., both systems are metallic, in agreement with findings from ARPES for the Si(553)-Au [27], STM [9], and theory [8]. However, it is at variance with the ARPES data for the $\mathrm{Si}(775)-\mathrm{Au}$ [27] for reasons still to be explored.

In the direction along the wires, clear loss features are observed, which shift to higher loss energies with increasing scattering angles, i.e., with increasing $k_{\|}$. In the $k_{\perp}$ direction, however, i.e., in the direction across the wires, no dispersing 
(a)

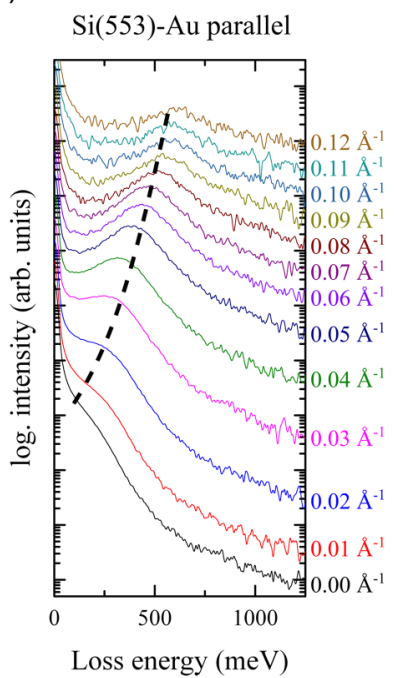

(b)

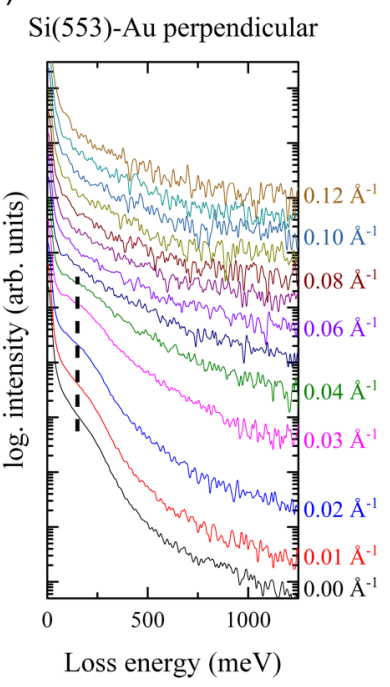

FIG. 2. Electron energy loss spectra (primary energy $20 \mathrm{eV}$ ) of $\mathrm{Au}$ chains grown on $\mathrm{Si}(553)$ as a function of $k$ parallel and normal to the wires, as indicated.

mode is seen [see Fig. 2(b)]. These findings are indicative of the existence of $1 \mathrm{D}$ plasmons.

Each spectrum was accurately fitted by parametrizing the elastically scattered peak, the actual plasmon loss, and the Drude background, and by applying the same fitting routine to all spectra. Details about the fitting procedure can be found in the Supplemental Material and are also elaborated in the Appendix of Ref. [36].

We note that special care has to be taken to eliminate water and hydrogen from the background gas. Under suboptimal vacuum conditions, disorder was observed in the system, increasing with time. The result is the (electronic) breakup of the Au chains, as is evident from the appearance of losses at finite energy and small $k_{\|}$whose loss energies increase with time. Such confined states were seen recently also for $\mathrm{Ag} / \mathrm{Si}(557)[36,37]$.

The dispersion curves along the wires resulting from the loss maxima of Fig. 2 are shown in Fig. 3. Both systems seem to be essentially quasi-1D systems, and therefore should be compared with existing 1D plasmon theory [17,18,25,31]. Common to all theoretical approaches is the use of a nearly free electron gas and various approximations for correlations, in the simplest case the random phase approximation (RPA). With this type of approach, a quantitative fit turned out to be possible with the model of coupled wires sitting in a periodic array of square potentials at distance $d[31,32]$. At small $k_{\|}$, the dispersion is given by

$$
\begin{aligned}
E= & \hbar \sqrt{\frac{4 n e^{2}}{(1+\epsilon) \epsilon_{0} m^{*} a^{2}}} k_{\|} a_{0} \\
& \times \sqrt{K_{0}\left(\frac{k_{\|} a}{2 \sqrt{2}}\right)+2 \sum_{l=1}^{L} K_{0}\left(k_{\|} l d\right) \cos \left(k_{\perp} l d\right),}
\end{aligned}
$$

where the first term of the product contains the electronic and structural properties of a single wire, and the second the intrawire (first term under the square root) and the interwire

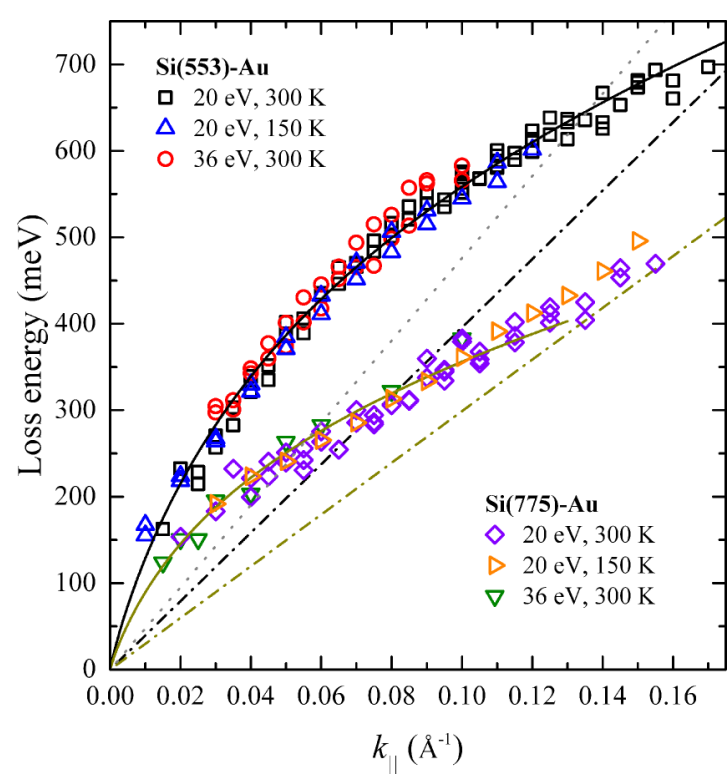

FIG. 3. Plasmon dispersion for Au quantum wires grown on $\mathrm{Si}(553)$ and on $\mathrm{Si}(775)$. Lines are fits according to Eq. (1). Dasheddotted lines: First terms of Eq. (1) for both systems. Dotted line: Same for $\mathrm{Si}(557)-\mathrm{Au}$; fitted data from Ref. [28].

interaction. $n$ is the electron density per unit length, $e$ the elementary charge, and $m^{\star}$ the effective mass. $\epsilon$ is the dielectric function of Si as partially embedding medium. $K_{0}$ are modified Bessel functions of zeroth order and the second kind, and $k_{\perp}$ is the momentum normal to the wires. If $a$ (the effective wire width) is set equal to $a_{0}$ (a constant for normalization), Eq. (1) corresponds to the original formula given in Refs. [31,32], which, however, turns out not to describe our findings. The ratio $a_{0} / a$ accounts both for differences in structural motifs and effective wire widths of a single wire, and is the only free parameter in Eq. (1). In the array of square potentials the first term under the second square root accounts for the self-interaction of a single wire, whereas the second term describes the interaction between different wires at multiple distances of $d$.

$n$ and $m^{\star}$ for the present systems were directly derived from ARPES data [27], i.e., from the occupied band structure of the Au-modified surface states. We also use their surface band notation. The systems investigated here are characterized by two surface bands, an upper $S_{1}$ and lower $S_{2}$, crossing the Fermi level [27,30]. As we see a metallic behavior of the $\mathrm{Si}(775)-\mathrm{Au}$ system, we assume its bands also cross the Fermi level for a clean sample at room temperature. The ratio $n / m^{\star}$ for both bands is identical within error bars. This means that the plasmons of these bands are degenerate and cannot be separated experimentally. As a consequence, only a single dispersion curve is expected to be seen for both systems with the electron density corresponding to a single band, in agreement with our findings.

The fits are shown in Fig. 3 together with the data. An explicit dependence of initial slope and shape of dispersion on structural motifs and on terrace widths is found, which is not described by existing 1D theories, as we will now demonstrate by concentrating on the first term in Eq. (1). 


\section{T. LICHTENSTEIN et al.}

Comparing first $\mathrm{Si}(553)$-Au and $\mathrm{Si}(775)-\mathrm{Au}$, both of which have the Au double chain but different terrace widths, this first term of Eq. (1) differs by a factor of 1.4 [after correction for small differences in the density of states (DOS)], as indicated by the dashed-dotted lines in Fig. 3, i.e., it scales with the inverse of the terrace width $d(21.3 \AA / 14.8 \AA=1.43)$.

$\mathrm{Si}(775)-\mathrm{Au}$ and $\mathrm{Si}(557)-\mathrm{Au}$ [28], on the other hand, have the same terrace widths (within $10 \%, 19.2$ vs $21.3 \AA$ ), but double and single $\mathrm{Au}$ chains per terrace, respectively. As seen from ARPES data [27], the 1D electron density of Si(775)-Au in the $S_{2}$ band is higher by $20 \%$ than for $\mathrm{Si}(557)-\mathrm{Au}$, whereas the effective masses are virtually the same. However, when fitting the published data of Ref. [28] for Si(557)-Au to Eq. (1), it turns out that its first term is a factor of 1.6 larger for $\mathrm{Si}(557)-\mathrm{Au}$ than that of Si(775)-Au. Taking the differences in $n$ and the $d$ dependence from above for the two systems, the effective width $a$, as suggested in Eq. (1), has to be reduced effectively by roughly a factor of 2 for $\mathrm{Si}(557)$-Au compared to $\mathrm{Si}(775)-\mathrm{Au}$. In other words, not only does the periodicity, given by the wire distances $d$, influence the dispersion directly, but also the internal $2 D$ distribution of electron density within each wire plays an important role in the plasmon dispersion. Since for these narrow structures and the given $k_{F}$ from ARPES only the lowest subband of a quantum well is occupied, combined excitations such as intersubband plasmon excitations can be ruled out.

These findings can be summarized by

$$
\omega_{p} \propto \sqrt{\frac{n a_{0}^{2}}{m^{\star} a^{2}}},
$$

with $a=\gamma d$, where $\gamma<1$ is determined by the internal lateral distribution of the electron density in each wire. This means that even in the case of purely $1 \mathrm{D}$ plasmonic dispersion, there is a crossover to $2 \mathrm{D}$, since the width of a wire on the atomic scale and the internal electronic distribution within the very wire enter directly the slope of plasmonic dispersion, which cannot be treated as a correction to 1D properties.

With respect to coupling between the wires, we obtained the best fit when the sum in Eq. (1) under the square root is truncated after the second term $(L=2)$, an indication of a finite range of interaction. This result may not be quantitative, since the model of Eq. (1) neglects damping and dephasing between wires. On the other hand, this analysis clearly demonstrates that the array of 1D plasmons is coupled.

Our plasmon analysis suggests that the internal modulation of the relevant electron density within each terrace, i.e., its finite extension perpendicular to the chain direction, plays a key role despite the otherwise 1D characteristics of these chain systems. Although this electronic modulation may not be exactly the same in the collectively excited state, such a modulation is indeed seen in scanning tunneling spectroscopy (STS) close to the Fermi level, using the Si(553)-Au system as a test system. This corroborates our suggestions from above. The combination of STM and STS (Fig. 4) not only shows the modulation, but it demonstrates that the highest density of states (DOS) is indeed located at the gold chains. The full width at half maximum (FWHM) of this modulation [see Fig. 4(b)] is $6.5 \pm 0.5 \AA$, i.e., it is close to the geometric width of a double gold chain on a $\mathrm{Si}(111)$ terrace. Although the amplitude of
PHYSICAL REVIEW B 93, 161408(R) (2016)

(a)

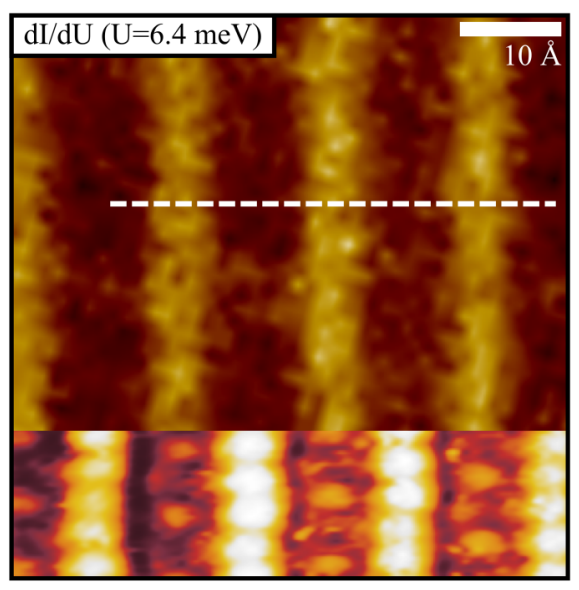

(b)

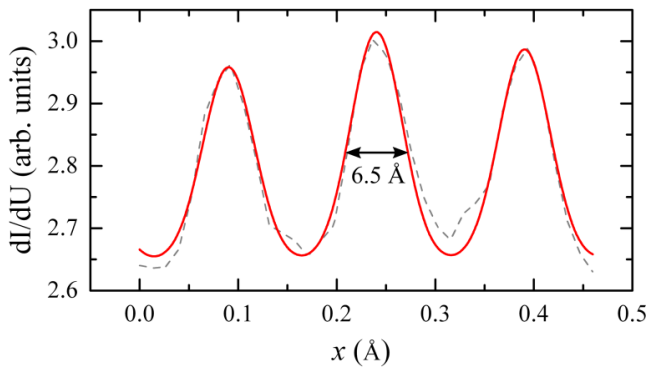

FIG. 4. (a) Combined image of tunneling microscopy and spectroscopy of $\mathrm{Au}$ on $\mathrm{Si}(553)$. Bottom: Topography image $(U=0.1 \mathrm{~V}$, $50 \mathrm{pA}$ ) with Si edge (bright) and period doubled Au chains, very similar to $\mathrm{Si}(775)$-Au (see Fig. 1). Top: $d I / d U$ map of the same sample area recorded with the lock-in technique $\left(U_{\text {mod }}=10 \mathrm{mV}\right)$ displayed close to $E_{F}\left(U_{\mathrm{dc}}=+6.4 \mathrm{meV}\right)$. The density of states (DOS) is significantly enhanced at the Au chain position. (b) Line scan through the $d I / d U$ map from above normal to the steps indicated by the white dashed line; the fit is given by the red curve.

this modulation is only around $10 \%$, it clearly demonstrates that also for the plasmon excitation this electronic density modulation must be relevant. If the plasmon of the lowest subband is also confined to the gold double chain, a comparable width is expected. Indeed, using Eq. (1) and setting $a_{0}$ to the separation of atomic rows on the $\mathrm{Si}(111)$ terraces of $3.32 \AA$, best fits of the measured dispersion curves are obtained with values of $a=7.5 \AA$ for $\mathrm{Si}(553)-\mathrm{Au}, 10.2 \AA$ for $\mathrm{Si}(775)-\mathrm{Au}$, and $5.9 \AA$ for $\mathrm{Si}(557)-\mathrm{Au}$. The latter value was obtained by neglecting coupling between wires in the fit. These values suggest that confinement of quasi-1D plasmons is related, but is not simply determined by the geometric width of the electronic ground state, and that both the structural motif and the terrace width play a crucial role.

Summarizing, we investigated plasmonic excitations in the narrowest quasi-1D systems experimentally possible, i.e., in arrays of atomic metallic wires formed by single and double Au chains in a vicinal $\mathrm{Si}(111)$ surface at various step densities. While only a $1 \mathrm{D}$ dispersing plasmon mode along the wires was found, the slope of the dispersion explicitly depends on the charge distribution within each miniterrace and on distance between wires, even for identical $1 \mathrm{D}$ ratios $n / m^{\star}$, thus superimposing $2 \mathrm{D}$ properties onto the $1 \mathrm{D}$ dispersion. While these findings require extensions of $1 \mathrm{D}$ plasmon theory, this 
dimensional crossover leads to further possibilities for tuning 1D plasmon dispersions.
Financial support by the Deutsche Forschungsgemeinschaft through FOR1700 is gratefully acknowledged.
[1] S. Kagoshima, H. Nagasawa, and T. Sambongi, OneDimensional Conductors, Springer Series in Solid State Sciences No. 72 (Springer, Berlin, 1988).

[2] G. Grüner, Rev. Mod. Phys. 60, 1129 (1988).

[3] J. M. Luttinger, J. Math. Phys. (N.Y.) 4, 1154 (1963).

[4] K. Schönhammer, in Strong Interactions in Low Dimensions, edited by D. Baeriswyl and L. Degiorgi (Kluwer Academic, Dordrecht, 2004), pp. 93-136.

[5] T. Giamarchi, Quantum Physics in One Dimension (Oxford University Press, Oxford, UK, 2007).

[6] C. Blumenstein, J. Schäfer, S. Mietke, S. Meyer, A. Dollinger, M. Lochner, X. Y. Cui, L. Patthey, R. Matzdorf, and R. Claessen, Nat. Phys. 7, 776 (2011).

[7] H. Weitering, Nat. Phys. 7, 744 (2011).

[8] S. C. Erwin and F. J. Himpsel, Nat. Commun. 1, 58 (2010).

[9] J. Aulbach, J. Schäfer, S. C. Erwin, S. Meyer, C. Loho, J. Settelein, and R. Claessen, Phys. Rev. Lett. 111, 137203 (2013).

[10] C. Brand, H. Pfnür, G. Landolt, S. Muff, J.-H. Dil, T. Das, and C. Tegenkamp, Nat. Commun. 6, 8118 (2015).

[11] P. C. Snijders and H. H. Weitering, Rev. Mod. Phys. 82, 307 (2010).

[12] B. Schwarz, P. Reininger, D. Ristanić, H. Detz, A. M. Andrews, W. Schrenk, and G. Strasser, Nat. Commun. 5, 4085 (2014).

[13] H. A. Atwater and A. Polman, Nat. Mater. 9, 205 (2010).

[14] C. Ayala-Orozco et al., ACS Nano 8, 6372 (2014).

[15] T. Nagao, G. Han, C. V. Hoang, J.-S. Wi, A. Pucci, D. Weber, F. Neubrech, V. M. Silkin, D. Enders, O. Saito, and M. Rana, Sci. Technol. Adv. Mater. 11, 054506 (2011).

[16] L. Vattuone, M. Smerieri, T. Langer, C. Tegenkamp, H. Pfnür, V. M. Silkin, E. V. Chulkov, P. M. Echenique, and M. Rocca, Phys. Rev. Lett. 110, 127405 (2013).

[17] S. Das Sarma and W.-y. Lai, Phys. Rev. B 32, 1401 (1985).

[18] T. Nagao, S. Yaginuma, T. Inaoka, T. Sakurai, and D. Jeon, J. Phys. Soc. Jpn. 76, 114714 (2007).
[19] E. P. Rugeramigabo, C. Tegenkamp, H. Pfnür, T. Inaoka, and T. Nagao, Phys. Rev. B 81, 165407 (2010).

[20] U. Krieg, C. Brand, C. Tegenkamp, and H. Pfnür, J. Phys.: Condens. Matter 25, 014013 (2013).

[21] M. Smerieri, L. Vattuone, L. Savio, T. Langer, C. Tegenkamp, H. Pfnür, V. M. Silkin, and M. Rocca, Phys. Rev. Lett. 113, 186804 (2014).

[22] J. Lindhard, Mat.-Fys. Medd. 28, 8 (1954).

[23] F. Stern, Phys. Rev. Lett. 18, 546 (1967).

[24] K. Singwi, M. Tosi, R. Land, and A. Sjölander, Phys. Rev. 176, 589 (1968).

[25] T. Inaoka and T. Nagao, Mater. Trans. 48, 718 (2007).

[26] J. Aulbach, S. Erwin, R. Claessen, and J. Schäfer, Nano Lett. 16, 2698 (2016).

[27] J. N. Crain, J. L. McChesney, F. Zheng, M. C. Gallagher, P. C. Snijders, M. Bissen, C. Gundelach, S. C. Erwin, and F. J. Himpsel, Phys. Rev. B 69, 125401 (2004).

[28] T. Nagao, S. Yaginuma, T. Inaoka, and T. Sakurai, Phys. Rev. Lett. 97, 116802 (2006)

[29] M. Krawiec, Phys. Rev. B 81, 115436 (2010).

[30] I. Barke, F. Zheng, T. K. Rügheimer, and F. J. Himpsel, Phys. Rev. Lett. 97, 226405 (2006).

[31] Q. Li and S. Das Sarma, Phys. Rev. B 41, 10268 (1990).

[32] S. Das Sarma and E. H. Hwang, Phys. Rev. B 54, 1936 (1996).

[33] H. Claus, A. Büssenschütt, and M. Henzler, Rev. Sci. Instrum. 63, 2195 (1992).

[34] G. Sauerbrey, Z. Phys. 155, 206 (1959).

[35] See Supplemental Material at http://link.aps.org/supplemental/ 10.1103/PhysRevB.93.161408 for (a) loss spectra of $\mathrm{Si}(775)$ $\mathrm{Au},(\mathrm{b})$ more details of fitting procedure, (c) loss intensities as a function of $k_{\|}$.

[36] U. Krieg, T. Lichtenstein, C. Brand, C. Tegenkamp, and H. Pfnür, New J. Phys. 17, 043062 (2015).

[37] U. Krieg, Y. Zhang, C. Tegenkamp, and H. Pfnür, New J. Phys. 16, 043007 (2014). 